\title{
A data-driven approach to local gravity field modelling using spherical radial basis functions
}

\author{
R. Klees · R. Tenzer - I. Prutkin - T. Wittwer
}

Received: 18 April 2007 / Accepted: 1 November 2007 / Published online: 2 February 2008

(C) The Author(s) 2008

\begin{abstract}
We propose a methodology for local gravity field modelling from gravity data using spherical radial basis functions. The methodology comprises two steps: in step 1, gravity data (gravity anomalies and/or gravity disturbances) are used to estimate the disturbing potential using least-squares techniques. The latter is represented as a linear combination of spherical radial basis functions (SRBFs). A data-adaptive strategy is used to select the optimal number, location, and depths of the SRBFs using generalized cross validation. Variance component estimation is used to determine the optimal regularization parameter and to properly weight the different data sets. In the second step, the gravimetric height anomalies are combined with observed differences between global positioning system (GPS) ellipsoidal heights and normal heights. The data combination is written as the solution of a Cauchy boundary-value problem for the Laplace equation. This allows removal of the non-uniqueness of the problem of local gravity field modelling from terrestrial gravity data. At the same time, existing systematic distortions in the gravimetric and geometric height anomalies are also absorbed into the combination. The approach is used to compute a height reference surface for the Netherlands. The solution is compared with NLGEO2004, the official Dutch height reference surface, which has been computed using the same data but a Stokes-based approach with kernel modification and a geometric six-parameter "corrector surface" to fit the gravimetric solution to the GPS-levelling points. A direct comparison of both height reference surfaces shows an RMS difference of $0.6 \mathrm{~cm}$; the maximum difference is $2.1 \mathrm{~cm}$. A test at
\end{abstract}

R. Klees $(\varangle) \cdot$ R. Tenzer $\cdot$ I. Prutkin $\cdot$ T. Wittwer

Delft Institute of Earth Observation and Space Systems (DEOS), Delft University of Technology, Kluyverweg 1,

2629 HS Delft, The Netherlands

e-mail: r.klees@tudelft.nl independent GPS-levelling control points, confirms that our solution is in no way inferior to NLGEO2004.

Keywords Local gravity field modelling - Spherical radial basisfunctions · GPS-levelling data · Quasi-geoid

\section{Introduction}

Spherical radial basis functions (SRBFs) are being used extensively in gravity field modelling. Examples are the point-mass kernel (e.g. Heikkinen 1981; Barthelmes 1986, 1988; Vermeer 1982, 1983, 1984, 1995), the radial multipoles (e.g. Marchenko 1998; Marchenko et al. 2001), the Blackman functions of Blackman and Tukey (1958) (Schmidt et al. 2004, 2005a, 2007), and the Poisson wavelets of Holschneider et al. (2003) (e.g. Chambodut et al. 2005; Panet et al. 2006; Klees and Wittwer 2007b), see also Holschneider and Iglewska-Nowak (2007). Other examples include the Dirac approach by Bjerhammar (1976), the scaling functions used in Freeden et al. (1998), spherical spline functions (e.g. Freeden and Reuter 1983; Freeden et al. 1997; Kusche et al. 1998), and the Kelvin-transformed reproducing kernels used in least-squares collocation (LSC) (e.g. Lelgemann 1981; Tscherning 1986; Lelgemann and Marchenko 2001).

To our opinion, the reason for the popularity of SRBFs in gravity field modelling is due to the following properties: (1) when located inside the masses, the harmonicity outside the masses in guaranteed; (2) they lead to simple functional models for all relevant gravity field functionals; (3) they are suited for global and local gravity field modelling; (4) they allow for local refinements of a spherical harmonic representation of the global field; and (5) they can be easily adapted to the data distribution and the signal variation. 
When using SRBFs in gravity field modelling, the user has to make a number of choices: (1) the type of the SRBF; (2) the axis or horizontal position of the SRBF; (3) the bandwidth of the SRBF; and (4) the number of SRBFs. The quality of the gravity field model and/or the numerical efficiency may depend on these choices. For instance, too many selected SRBFs may cause overfitting; a bandwidth, which is too large for a given set SRBFs, causes instabilities as the significant support of neighboured SRBFs overlap too much, which leads to similar columns in the design matrix; a bandwidth, which is too small for a given number of SRBFs may lead to a nice fit of the data to the model, but the fit at a set of independent control points may be worse.

Little is known about the influence of the type of SRBF on the estimated gravity field. In a recent paper by Tenzer and Klees (2007), the performance of different SRBFs in regional gravity field modelling from gravity anomaly data was investigated. No significant differences were found provided that the bandwidths of the SRBFs were chosen properly. Similar experiences are reported by Wittwer (personal communication, 2007) for the processing of data from the Gravity Field and Climate Experiment (GRACE) mission.

Among the approaches commonly used to fix the centres of the SRBFs are hierarchical or non-hierarchical subdivision schemes, e.g. based on a triangulation of an icosahedron (e.g. Eicker et al. 2004), homogeneous point distributions, derived from the theory of numerical integration of spherical functions (e.g. Schmidt et al. 2004), or equal angular grids (e.g. Klees and Wittwer 2007a). Alternatively, some approaches identify the centres of the SRBFs with those of the data points (e.g. Heikkinen 1981; Vermeer 1982, 1983, 1984) or a subset of the data points (e.g. Marchenko et al. 2001). More sophisticated approaches apply (non-linear) optimization algorithms, to fix centres and bandwidths simultaneously (e.g. Barthelmes 1986). Sometimes, data are first gridded using some interpolation or approximation algorithm, and the SRBFs are placed below the grid points. Then, fast (frequency domain) methods are used to estimate the unknown gravity field parameters (e.g. Bottoni and Barzaghi 1993; Sanso and Tscherning 2003).

Many studies have been conducted to find the optimal bandwidths of the SRBFs, which for the majority of SRBFs used in gravity field modelling is equivalent with the choice of the depths of the SRBFs below the Bjerhammar sphere. Often, the bandwidth is selected by "trial-and-error" or based on empirically found relations with the data spacing or the gravity anomaly autocovariance function (e.g. Blaha et al. 1986; Hardy and Göpfert 1975; Heikkinen 1981; Sünkel 1981). More advanced strategies involve generalized cross validation (GCV) techniques (e.g. Klees and Wittwer $2007 a, b)$ or the adaptation of the shape of the SRBF to the local signal covariance function (Marchenko et al. 2001).
Sometimes, SRBF grids with different spacing on several layers at various depth are used (e.g. Reilly and Herbrechtsmeier 1978; Heikkinen 1981; Vermeer 1982, 1983 , 1984).

Some authors determine the centres and bandwidths of SRBFs directly, based on information contained in the data. For instance, Barthelmes (1986) designed a fairly stable algorithm for the optimization of point masses with free positions, which solves essentially a non-linear optimization problem with four parameters per point mass (three parameters to describe the position in 3D space and one parameter to fix the magnitude of the point mass). Optimization of the 3D positions of SRBFs may significantly reduce the number of point masses needed to approximate the data, which is an advantage for gravity field synthesis. Moreover, it prevents instability of the estimation process. However, the computational complexity of the gravity field analysis is significant.

An alternative to the method of Barthelmes (1986) has been developed by Marchenko (1998) (see also Marchenko et al. 2001; Marchenko 2003). He uses higher-order radial multipoles and locates them below the data points. An optimization algorithm (called sequential multipole algorithm $S M A$ ) reduces the number of radial multipoles by exploiting the (residual) signal at the data points (i.e. a multipole is not necessarily assigned to each data point) and, at the same time, selects the order of the radial multipole and the depth below the data point. The latter two parameters are fixed using the gravity anomaly covariance function in the neighbourhood of the data point. Depth and shape of the radial multipoles may differ from point to point. In that way, the number of radial multipoles can be reduced significantly. At the same time, the condition number of the normal equations improves. Marchenko et al. (2001) report comparable accuracies as provided by LSC, but with a number of basis functions that is about $70 \%$ less than the number of data points.

Klees and Wittwer (2007a) developed a data-adaptive strategy to select the centres and bandwidths of the SRBFs, which has been designed for heterogeneous data distributions (i.e. data sets with local concentration and gaps). Centres and bandwidths are selected as function of the data distribution and the signal variation using GCV techniques. This approach allows for a significant reduction of the number of SRBFs for areas with small spatial signal variations.

The subject of this paper is the development and application of a data-adaptive strategy for local gravity field modelling using a SRBF representation of the residual disturbing potential. The approach differs from what has been proposed so far in literature with respect to a number of aspects:

1. the number and axes of the SRBFs are selected automatically from the data using a two-step procedure to improve the numerical efficiency; 
2. the bandwidths of the SRBFs are selected from the data using GCV;

3. weight factors of individual observation groups and the regularization parameter are determined using VCE techniques;

4. various terrestrial data types such as gravity anomalies, gravity disturbances, and height anomalies can be dealt with;

5. the combination of gravimetric data with GPS-levelling data is done according to the method of Prutkin and Klees (2007);

6. a penalized least-squares (LS) techniques is applied to estimate the gravity field parameters;

7. the algorithms are designed to allow processing of large data sets.

The integration of these elements into one approach provides a numerically very efficient and accurate description of the local gravity field and paves the way for an integrated approach to regional gravity field modelling from a combination of terrestrial data with airborne gravimetry data and satellite gravity data.

The paper is organized as follows: in Sect. 2, SRBFs are defined. In Sect. 3, the mathematical model and the estimation principle are specified. We prefer a penalized LS principle to estimate the unknown gravity field parameters. A sketch of the overall data processing strategy is given in Sect. 4. In Sect. 5, we describe a two-step procedure to select the centres of the SRBFs in a numerically efficient way, comprising a coarse grid step and a local refinement step. Section 6 is devoted to the selection of the optimal bandwidth using generalized cross validation (GCV) techniques. In Sect. 7, we address the problem of optimal data weighting of individual observation groups and the optimal choice of the regularization parameter. For both tasks, we propose to use variance component estimation (VCE) techniques. In Sect. 8, the combination of gravity data with GPSlevelling data is addressed. We apply the methodology of Prutkin and Klees (2007). In Sect. 9, the developed method is applied to real data in the Netherlands. The results are compared with the official Dutch height reference surface NLGEO2004 (Crombaghs and de Bruijne 2004), which has been computed using a Stokes's approach with kernel modification and a bi-quadratic "corrector surface" to model differences between geometric and gravimetric height anomalies. Finally, in Sect. 10, we provide a summary of the research and draw conclusions.

\section{Spherical radial basis functions}

We denote by $\sigma_{R}$ the surface of the sphere of radius $R$, which is located completely inside the topographic masses
(Bjerhammar sphere), $\sigma_{R}=\left\{\left(x_{1}, x_{2}, x_{3}\right): x_{1}^{2}+x_{2}^{2}+x_{3}^{2}=\right.$ $\left.R^{2}\right\}$. We denote by Int $\sigma_{R}$ the interior and by Ext $\sigma_{R}$ the exterior. We consider two points $x, y \in \mathbb{R}^{3}, y \neq 0$, with $x=\left(x_{1}, x_{2}, x_{3}\right)^{\mathrm{T}}$ and $y=\left(y_{1}, y_{2}, y_{3}\right)^{\mathrm{T}}$. We define the exterior SRBF at pole position $y$ evaluated at $x$ through

$$
\begin{aligned}
\Psi(x, y)= & \sum_{l=0}^{\infty} \psi_{l}(y) \frac{2 l+1}{4 \pi R^{2}}\left(\frac{R}{|x|}\right)^{l+1} P_{l}\left(\hat{x}^{\mathrm{T}} \hat{y}\right), \\
& x \in \overline{\operatorname{Ext} \sigma_{R}}, \quad y \in \operatorname{Int} \sigma_{R},
\end{aligned}
$$

where $P_{l}$ is the Legendre polynomial of degree $l$ and $\hat{x}=\frac{x}{|x|}$ and $\hat{y}=\frac{y}{|y|}$ are unit vectors in the direction $x$ and $y$, respectively. The exterior SRBF is a harmonic function in Ext $\sigma_{R}$. It is a zonal function, that is, it is rotational symmetric around the axis $\hat{y}$. The point $y$ is called the centre (sometimes also the pole or the nodal point) of the SRBF. Different choices of $\left\{\psi_{l}\right\}$ generate different types of SRBFs. Table 1 shows the Legendre coefficients for a number of SRBFs used in gravity field modelling and, if available, the analytical representation.

\section{Mathematical model and estimation principle}

We consider a residual gravity field, which is obtained after the contributions of a global gravity field model complete to degree $L$ and of the topography computed from a digital terrain model and a mean value of the crustal density or a digital density model have been subtracted. The residual disturbing potential, $T$, is expressed as a linear combination of $N$ basis functions $\left\{\Psi_{n}(x): n=1, \ldots, N\right\}$,

$T(x)=\sum_{n=1}^{N} \beta_{n} \Psi_{n}(x)$,

where the real numbers $\beta_{n}$ are the coefficients to be determined from data, and

$\Psi_{n}(x)=\Psi\left(x, y_{n}\right)$,

with $\Psi(x, y)$ from Eq. (1). We work in the framework of the Runge-Krarup theorem (Krarup 1969). That is, $T$ is considered as a member of the function space of regular harmonic functions outside the Bjerhammar sphere with radius $R$ located inside the topographic masses, and it is taken as an approximation of the true residual disturbing potential at points on and outside the Earth's surface. The summation in Eq. (1) may start with an index $l_{\min }>0$. Often, $l_{\min }=L+1$ is chosen, as one assumes that the residual disturbing potential does not contain enough signal below degree $L+1$ or that the disturbing potential is represented by a series expansion into spherical harmonics up to degree $L$ and a finite number of SRBFs (e.g. Schmidt et al. 2005b). From numerous numerical experiments with terrestrial gravity data, we 
Table 1 Legendre coefficients and analytical expressions for several SRBFs often used in gravity field modelling

\begin{tabular}{|c|c|c|}
\hline Point mass kernel & $\psi_{l}=\frac{4 \pi R}{2 l+1} \lambda^{l}, \quad \lambda=\frac{|y|}{R}$ & $\Psi(x, y)=\frac{1}{|x-y|}$ \\
\hline Radial multipole of order $m$ & $\psi_{l}=\left\{\begin{array}{ll}\left(\begin{array}{l}l \\
m\end{array}\right) \frac{\lambda^{l-m}}{2 l+1} & l \geq m \\
0 & l<m\end{array}, \lambda=\frac{|y|}{R}\right.$ & $\Psi(x, y)=\frac{1}{m !}\left(\frac{\partial}{\partial|y|}\right)^{m} \frac{1}{|x-y|}$ \\
\hline Poisson wavelet of order $m$ & $\psi_{l}=l^{m} \lambda^{l}, \quad \lambda=\frac{|y|}{R}$ & $\begin{array}{l}\Psi(x, y)=\frac{1}{4 \pi R^{2}}\left(2 \chi_{m+1}+\chi_{m}\right) \\
\chi_{m}=\left(|y| \frac{\partial}{\partial|y|}\right)^{m} \frac{1}{|x-y|}\end{array}$ \\
\hline Dirac approach of Bjerhammar & $\psi_{l}= \begin{cases}\frac{4 \pi R^{3}}{l-1} & l \geq 2 \\
0 & l=0,1\end{cases}$ & \\
\hline Poisson kernel & $\psi_{l}=\lambda^{l}$ & $\Psi(x, y)=\frac{1}{4 \pi R} \frac{|x|^{2}-|y|^{2}}{|x-y|^{3}}$ \\
\hline
\end{tabular}

found that $l_{\min }=0$ works fine even if the latest Gravity Field and Climate Experiment (GRACE)-based global gravity field model has been subtracted. The reason is that in local gravity field modelling, the depth of the SRBFs is shallow (a few kilometres) so that the sum of the contribution of the low-degree Legendre coefficients cancel, i.e. they have no significant influence on the shape of the SRBF.

The objective of local gravity field modelling is to determine the coefficients $\left\{\beta_{n}: n=1, \ldots, N\right\}$ from functionals $\left\{L_{i} T: i=1, \ldots, I\right\}$ of the residual disturbing potential $T$, which have been measured at the Earth's surface. Examples of linear functionals used in local gravity field modelling are (residual) gravity anomalies $\Delta g$, (residual) gravity disturbances $\delta g$, and (residual) height anomalies $\zeta$. The latter are usually computed as differences between global positioning system (GPS) ellipsoidal heights and normal heights. We assume that, after linearization and spherical approximation, these functionals are related to the (residual) disturbing potential as

$$
\begin{aligned}
\Delta g(x) & =-\frac{2}{|x|} T(x)-\frac{\partial T(x)}{\partial|x|}, \\
\delta g(x) & =-\frac{\partial T(x)}{\partial|x|}, \\
\zeta(x) & =\frac{T(x)}{\gamma\left(x^{\prime}\right)},
\end{aligned}
$$

where $\gamma$ is normal gravity and $x^{\prime}$ is the point on the telluroid associated with the surface point $x$ by a telluroid mapping.

Depending on the data quality, the quantities on the lefthand side of Eqs. (4), (5), and (6) may be corrected for e.g. linearization and spherical approximation errors (e.g. Heck and Seitz 1993, 2003).

Assuming that $I$ linear functionals $l_{i}=L_{i} T$ have been observed, we obtain the system of observation equations

$l_{i}-\varepsilon_{i}=\sum_{n=1}^{N} \beta_{n} L_{i} \Psi\left(x, y_{n}\right), \quad I \geq N$.

For the (residual) gravity anomaly, the (residual) gravity disturbance, and the (residual) height-anomaly, at the point $\xi_{i}$, we have

$$
\begin{aligned}
& L_{i} T=\Delta g\left(\xi_{i}\right)=\sum_{n=1}^{N} \beta_{n} D_{\Delta g} \Psi\left(\xi_{i}, y_{n}\right), \\
& L_{i} T=\delta g\left(\xi_{i}\right)=\sum_{n=1}^{N} \beta_{n} D_{\delta g} \Psi\left(\xi_{i}, y_{n}\right), \\
& L_{i} T=\zeta\left(\xi_{i}\right)=\sum_{n=1}^{N} \beta_{n} D_{\zeta} \Psi\left(\xi_{i}, y_{n}\right),
\end{aligned}
$$

where $D_{\Delta g}, D_{\delta g}$ and $D_{\zeta}$ is the gravity anomaly operator, the gravity disturbance operator, and the height-anomaly operator, respectively. In vector-matrix notation, Eq. (7) is rewritten as

$\mathbf{l}-\mathbf{e}=\mathbf{A} \mathbf{x}$

where $\mathbf{A}$ is the $I \times N$ design matrix with elements

$A_{i, n}=L_{i} \Psi\left(x, y_{n}\right), \quad i=1, \ldots, I, \quad n=1, \ldots, N$,

$\mathbf{x}$ is the $N \times 1$ vector of unknown parameters with elements

$x_{n}=\beta_{n}, \quad n=1, \ldots, N$,

and $\mathbf{e}$ is the $I \times 1$ vector of stochastic observation errors with expectation $E(\mathbf{e})=0$ and dispersion $D(\mathbf{e})=\mathbf{C}$.

We assume that the observation vector $\mathbf{l}$ consists of $P$ disjunct groups $\mathbf{l}_{p}$. The corresponding vectors of stochastic observation errors $\mathbf{e}_{p}$ may be uncorrelated, but elements of a particular vector $\mathbf{e}_{p}$ may be correlated. Then,

$\mathbf{C}=\left(\begin{array}{ccccc}\mathbf{C}_{1} & 0 & 0 & \ldots & 0 \\ 0 & \mathbf{C}_{2} & 0 & \ldots & 0 \\ \vdots & \vdots & \vdots & \ldots & \vdots \\ 0 & 0 & 0 & \ldots & \mathbf{C}_{p}\end{array}\right)$.

For instance, all gravity anomalies may form one observation group, all gravity disturbances may form another one, etc. One may also split the gravity anomaly data set into several observation groups, e.g. if one expects that the quality differs 
significantly. Then, Eq. (11) is written as

$$
\left(\begin{array}{c}
\mathbf{l}_{1} \\
\vdots \\
\mathbf{l}_{p}
\end{array}\right)-\left(\begin{array}{c}
\mathbf{e}_{1} \\
\vdots \\
\mathbf{e}_{p}
\end{array}\right)=\left(\begin{array}{c}
\mathbf{A}_{1} \\
\vdots \\
\mathbf{A}_{p}
\end{array}\right) \mathbf{x},
$$

where $\mathbf{A}_{p}$ is the design matrix of observation group $p$. The chosen estimation principle is a penalized LS principle: for some given $\alpha$ (the regularization parameter), we minimize the quadratic functional

$\Phi(\mathbf{x})=\|\mathbf{e}\|_{\mathbf{C}^{-1}}^{2}+\alpha J(T)$,

where

$\|\mathbf{e}\|_{\mathbf{C}^{-1}}^{2}=\sum_{p=1}^{P}\left\|\mathbf{e}_{p}\right\|_{\mathbf{C}_{p}^{-1}}^{2}$,

and

$J(T)=\|\mathbf{x}\|_{\mathbf{R}}^{2}$.

$\mathbf{R}$ is a positive definite (regularization) matrix. For a given regularization parameter $\alpha$ and given variance factors $\left\{\sigma_{p}^{2}\right.$ : $p=1, \ldots, P\}$, the minimum of the quadratic functional $\Phi(\mathbf{x})$ is attained for

$\hat{\mathbf{x}}=\mathbf{N}^{-1} \mathbf{h}$,

with

$\mathbf{N}=\sum_{p=1}^{P} \mathbf{N}_{p}+\alpha \mathbf{R}, \quad \mathbf{N}_{p}=\mathbf{A}_{p}^{T} \mathbf{C}_{p}^{-1} \mathbf{A}_{p}$,

and

$\mathbf{h}=\sum_{p=1}^{P} \mathbf{h}_{p}, \quad \mathbf{h}_{p}=\mathbf{A}_{p}^{T} \mathbf{C}_{p}^{-1} \mathbf{l}_{p}$.

How to determine $\alpha$ will be discussed in Sect. 7. Often, $\mathbf{R}=$ $\mathbf{I}_{N}$, where $\mathbf{I}_{N}$ is the $N \times N$ unit matrix.

\section{Sketch of the data processing strategy}

The data processing strategy consists of four steps; the first three steps are devoted exclusively to the processing of gravity data; the fourth step performs the combination of gravimetric height-anomalies (which is the result of steps 1-3) with GPS-levelling data.

Step 1. A spherical grid is defined and the grid points are used as the centres of the so-called coarse-grid SRBFs. A penalized LS solution (the so-called coarse-grid solution) is computed, and GCV is used to fix the bandwidth of the coarse-grid SRBFs, which is the same for all coarse-grid SRBFs.

Step 2. The LS residuals of step 1 are used to sequentially add additional SRBFs, which are located below data points.
Whether a SRBF is located below a data point depends on several criteria (Sect. 5). The optimal depth of each individual local-refinement SRBF is determined from the data in a neighbourhood of the candidate SRBF using GCV. The result of step 2 is a list of local refinement SRBFs with fixed centres and bandwidths.

Step 3. The coarse-grid SRBFs and the local-refinement SRBFs represent the complete parameterization of the disturbing potential. The coefficients of the SRBFs are determined by penalized LS using all gravity data. The centres and the bandwidths of the SRBFs are fixed to their values found in step 1 and step 2, respectively. The regularization parameter and the variance factors of individual observation groups are estimated using VCE (Sect. 7). Depending on a statistical analysis and a geographical plot of the LS residuals of step 3 , a new local refinement iteration can be performed to add additional SRBFs to the system. Then, the residuals of step 3 serve as input data. The solution obtained after finishing step 3 is the gravity field solution based on residual gravity anomaly and/or residual gravity disturbance data. We call this solution the gravimetric quasi-geoid and the corresponding height-anomalies, the gravimetric height-anomalies.

Step 4. From the estimated coefficients of step 3, gravimetric height-anomalies at the GPS-levelling points are predicted, and the differences between geometric heightanomalies (differences between GPS-ellipsoidal heights and normal heights) and gravimetric height-anomalies are formed; they are called height-anomaly differences. The height-anomaly differences are used to compute a function $\tilde{T}$, which is harmonic in the vicinity of the target area and has almost zero gravity anomaly signal over the target area (Prutkin and Klees 2007) (Sect. 8). From $\tilde{T}$, we can compute height-anomaly innovations $\tilde{\zeta}$ at any point inside the target area. The final height-anomaly is obtained as the sum of the gravimetric height-anomaly (i.e. the result after step 3) and the height-anomaly innovations.

\section{Choice of SRBF centres}

The coarse grid SRBFs represent a first-order parameterization of the disturbing potential. The spherical grid can be any homogeneous point distribution on the sphere, e.g. an equalangular grid or a grid generated by a subdivision scheme based on a cube, an icosahedron, etc. The mesh size of the grid, which has to be chosen by the user, must not be smaller than the mean distance between the data points. Otherwise, one takes the risk that too many SRBFs are used. Empirically, we found that the mesh size can be chosen at least $50 \%$ larger than the mean distance between the data points. Whether this is an appropriate choice, can be checked easily. For instance, if no local-refinement SRBFs are selected, it is an indication that the mesh size of the coarse grid is too 
fine. Then, one can select a coarser mesh size and repeat the local-refinement procedure. Vice versa, too many selected local-refinement SRBFs are an indication that the coarsegrid mesh size was chosen too coarse. Notice that a potential under-parameterization is automatically corrected for in step 2. Regularization $[\alpha \neq 0$ in Eq. (14)] is only applied if instabilities are observed. The goal of the first step is to obtain a coarse parameterization of the disturbing potential, which is further refined in step 2. The main benefit of step 1 is an improved numerical efficiency compared with a selection of the complete parameterization solely using step 2 .

The coarse-grid parameterization of the disturbing potential is refined by adding local-refinement SRBFs. A localrefinement SRBF differs from a coarse-grid SRBF in two respects: (1) it is always located below a data point, and (2) each local refinement SRBF has its own bandwidth. Whether or not a SRBF is located below a data point depends on the following criteria:

1. The magnitude of the residual at the candidate SRBF must be larger than some threshold $\tau_{1}$.

2. There must be at least $q$ data points in the neighbourhood of the candidate SRBF, with sufficiently large residuals. Sufficiently large means that the average absolute residual taken over the $q$ data points is larger than some threshold $\tau_{2}$. The neighbourhood of a candidate SRBF is defined as a spherical cap $\sigma_{c}$ centered at the candidate SRBF; the spherical radius of the cap is $\psi_{c}$.

3. The spherical distance between the candidate SRBF and any already selected SRBF (coarse grid or local refinement SRBF) must be larger than some minimum spherical distance $\psi_{\min }$.

The parameters $\tau_{1}, \tau_{2}, q, \psi_{c}$, and $\psi_{\min }$ are input parameters to be chosen by the user.

These three criteria aim at avoiding over-parameterization by adding too many SRBFs. For instance, criterion 2 should avoid that a SRBF is placed below an isolated, large residual, which is likely to be an artefact (e.g. a blunder) and not a gravity field signal. Criterion 3 should avoid that there is a local concentration of SRBFs, which do not model signal but neutralize themselves. Of course, placing a SRBF makes sense only if the magnitude of the residual is sufficiently large. This is guaranteed by criterion 1 and 2 . If more than one local refinement is performed, the parameters $\tau_{1}, \tau_{2}, q$, $\psi_{c}$, and $\psi_{\min }$ could be changed from one iteration to the next one.

The local refinement procedure is a four-step procedure (with "observations" in the local refinement procedure, we mean the residuals of the coarse-grid LS adjustment or the residuals of the previous local-refinement iteration):

Step 1. The largest "observation" is looked for. Then, it is checked whether criteria 1, 2, and 3 are fulfilled. If not, this point is removed from the list of candidate SRBFs and the second largest "observation" is selected.

Step 2. If the check has passed successfully, a local LS adjustment is performed: only the "observations" within the spherical cap $\sigma_{c}$ are used. The weight matrix is the unity matrix. The coefficient of the SRBF is the only unknown parameter; regularization is not needed. The optimal depth of the SRBF is determined using GCV.

Step 3. The residuals of the local LS adjustment are computed. They are taken as "observations" for the choice of the next candidate SRBF. Data outside the spherical cap $\sigma_{c}$ are not corrected for the contribution of the SRBF, i.e. they are left unchanged. This is justified because data outside the neighbourhood are almost unaffected by the selected SRBF. To ensure this, the minimum spherical distance $\psi_{\text {min }}$ should be a factor two to three larger than the correlation length of the selected local-refinement SRBF. This is a consistency check to be done by the user. On the other hand, deviations from this rule are not critical for the success of the local-refinement procedure.

Step 4. The corresponding data point is removed from the list of candidate SRBF locations and the procedure continues with the largest "observations" among the points left in the list of candidate SRBF locations. The local refinement procedure stops if the list of candidate SRBFs is empty.

\section{Choice of the SRBF bandwidths}

The most critical factor in optimal SRBF network design seems to be the proper choice of the bandwidth of the SRBFs. We use GCV (Golub et al. 1979) to select the optimal bandwidths of the SRBFs. Suppose the bandwidth of a SRBF is fixed by a single parameter $p$. For instance, the parameter $p$ is the depth of a SRBF below the Bjerhammar sphere for the Poisson kernel and the point mass kernel.

The principle of cross-validation is based on the leave-outone idea. Doing the LS adjustment without observation $l_{k}$, the corresponding leave-out-one solution vector $\hat{\mathbf{x}}^{(k)}$, which of course depends on the bandwidth of the SRBFs, i.e. $\hat{\mathbf{x}}^{(k)}=$ $\hat{\mathbf{x}}^{(k)}(p)$, can be used to predict the missing observation. It is natural to expect that a good choice of the bandwidth parameter $p$ results in a small difference (misfit) between the predicted value and the observed value. Doing this step-by-step for all observations, a good choice of the bandwidth should lead to a small misfit in average over all possible $l_{k}$. This so-called ordinary cross-validation parameter $\lambda_{\mathrm{cv}}$ is obtained as

$\lambda_{\mathrm{cv}}=\arg \min \left\{\frac{1}{I} \sum_{i=1}^{I} w_{i}\left(\hat{l}_{i}(p)-l_{i}\right)^{2}\right\}$, 
in which the weights are given as

$w_{i}=\frac{1}{\left(1-Q_{i i, p}\right)^{2}}$.

$\hat{l}_{i}(p)$ is the adjusted $i$ th observation, $\mathbf{Q}_{p}$ is the so-called influence matrix defined by $\mathbf{A} \hat{\mathbf{x}}=\mathbf{Q}_{p} \mathbf{l}, Q_{i i, p}$ is the $i$ th diagonal element of $\mathbf{Q}_{p}$, and $I=\sum_{j=1}^{J} I_{j}$ is the total number of observations. The generalized cross validation parameter $p_{\mathrm{gcv}}$ is obtained when the weights $w_{i}$ are replaced by their average value $\frac{1}{I}$ trace $\left(\mathbf{I}-\mathbf{Q}_{p}\right)$ :

$$
\begin{aligned}
p_{\mathrm{gcv}} & =\arg \min \left\{\frac{1}{I} \sum_{i=1}^{I} \frac{\left(\hat{l}_{i}(p)-l_{i}\right)^{2}}{\left(\frac{1}{I} \operatorname{trace}\left(\mathbf{I}-\mathbf{Q}_{p}\right)\right)^{2}}\right\} \\
& =\arg \min \left\{\sum_{i=1}^{I} \frac{I\left(\hat{l}_{i}(p)-l_{i}\right)^{2}}{\left(\operatorname{trace}\left(\mathbf{I}-\mathbf{Q}_{p}\right)\right)^{2}}\right\} .
\end{aligned}
$$

For a non-unity noise variance-covariance matrix $\mathbf{C}=$ $\sigma^{2} \mathbf{W}^{-1}$, the GCV estimate of $p$ reads

$p_{\mathrm{gcv}}=\arg \min \left\{\frac{I\|\mathbf{A} \hat{\mathbf{x}}(p)-\mathbf{I}\|_{\mathbf{W}}^{2}}{\left(\operatorname{trace}\left(\mathbf{I}-\mathbf{Q}_{p}\right)\right)^{2}}\right\}$.

\section{Regularization and observation group weighting}

We assume that every positive definite covariance matrix $\mathbf{C}_{p}=\sigma_{p}^{2} \mathbf{W}_{p}^{-1}$ is only given up to an unknown variance factor $\sigma_{p}^{2}$. The $P$ variance factors are estimated using VCE. This technique is also used to find the optimal regularization parameter. To do this, we rewrite Eq. (14) as

$\Phi(\mathbf{x})=\sum_{p=1}^{P}\left\|\mathbf{e}_{p}\right\|_{\mathbf{C}_{p}^{-1}}^{2}+\left\|\mathbf{e}_{P+1}\right\|_{\mathbf{C}_{P+1}^{-1}}^{2}$,

with the $(P+1)$ th "observation group"

$\mathbf{e}_{P+1}=\mathbf{I}_{N} \mathbf{x}, \quad D\left(\mathbf{e}_{P+1}\right)=\mathbf{C}_{P+1}=\frac{1}{\alpha} R^{-1}$.

Then, Eq. (17) is the classical (unconstrained) LS solution for $P+1$ observation groups with the $(P+1)$ th observation vector $\mathbf{l}_{P+1}=0$. Therefore, the determination of the regularization parameter is interpreted as the determination of the variance factor of an additional observation group. This observation group can be interpreted as directly observed unknown parameters with observation vector 0 and noise variance-covariance matrix $\frac{1}{\alpha} R^{-1}$. The relation between the regularization parameter and the variance factor $\sigma_{P+1}^{2}$ is $\alpha=$ $\frac{1}{\sigma_{P+1}^{2}}$.

We implemented the almost unbiased estimator (AUE) of the variance factors (e.g. Förstner 1979),

$\hat{\sigma}_{p}^{2}=\frac{\hat{\mathbf{e}}_{p}^{\mathrm{T}} \mathbf{W}_{p} \hat{\mathbf{e}}_{p}}{r_{p}}, \quad p=1, \ldots, P+1$, where $\hat{\mathbf{e}}_{p}$ is the vector of residuals of observation group $p$. The quantity

$r_{p}=I_{p}-\operatorname{trace}\left(\mathbf{N}^{-1} \mathbf{N}_{p}\right)$

is the group redundancy number. It is the difference of the number of observations of this group, $I_{p}$, with the trace of the observation group influence matrix $\mathbf{N}^{-1} \mathbf{N}_{p}$. This trace is a measure of the influence of the observations of group $p$ on the LS solution $\hat{\mathbf{x}}$. When this trace equals the number of unknowns, the LS estimate of the unknowns, $\hat{\mathbf{x}}$, is solely determined by this observation group. If the trace equals zero, the observation group does not contribute at all to the determination of the unknowns, i.e. it could be also excluded from the LS adjustment without changing the solution $\hat{\mathbf{x}}$. For instance, $r_{P+1}=I_{P+1}=N$. The sum of all group redundancy numbers equals the total redundancy, i.e. the difference between the number of observations and the number of unknown parameters.

The estimation of the variance factors is done iteratively, starting with some a priori values $\left\{\hat{\sigma}_{p, 0}^{2}: p=1, \ldots, P\right\}$. The LS solution $\hat{\mathbf{x}}$ is computed according to Eq. (17) using these a priori variance factors. The residuals of the LS adjustment are computed and improved values of the variance factors are obtained according to Eq. (25). In the next iteration, they are used to define new noise variance-covariance matrices $\mathbf{C}_{p}$, to set up the normal equations, etc. The procedure is repeated until convergence is achieved. We use

$\max _{p=1, \ldots, P+1} \frac{\left|\hat{\sigma}_{p, i}^{2}-\hat{\sigma}_{p, i-1}^{2}\right|}{\hat{\sigma}_{p, i}^{2}} \leq \tau$,

as a criterion for convergence, where $\tau$ is a threshold (e.g. $\tau=0.01$ ), and $\hat{\sigma}_{p, i}^{2}$ is the variance factor of observation group $p$ after iteration $i$. Notice that in the case of convergence, the AUE is equal to the ML estimator. To accelerate the computation of the group redundancy numbers, we implemented the Monte Carlo VCE technique of Koch and Kusche (2002), see also Kusche (2003).

\section{Combination of gravimetric and GPS-levelling data}

Height anomalies, derived from GPS-ellipsoidal heights and normal heights, have become a standard data type for local gravity field modelling. We call them geometric height anomalies to distinguish them from the gravimetric height anomalies, which are determined from terrestrial gravity anomalies and/or gravity disturbances. In practice, one often observes systematic differences between gravimetric and geometric height anomalies. These differences are often attributed to systematic errors in the data, e.g. systematic deformations of the national height system, different tide 
systems, multipath effects in GPS ellipsoidal heights, and long-wavelength errors in the gravimetric height anomalies.

In practice, one can parameterize these systematic differences between gravimetric and geometric height anomalies using a low-degree algebraic or trigonometric polynomial in latitude and longitude (or any other parameterization). Then, the parameters are estimated from the differences between both types of height anomalies using a standard LS technique. The parametric model may be extended by a stochastic signal term with given auto-covariance structure, which leads to a LSC problem with parameters. The final height reference surface is then obtained by adding the parametric surface (and the random signal part) to the gravimetrically determined quasi-geoid. This height reference surface does not need to represent the quasi-geoid anymore, but a surface that can best be used to transform GPS-ellipsoidal heights into normal heights.

There is, however, another contributor to the systematic differences between gravimetric and geometric height anomalies. It is caused by a non-trivial kernel of the operator that maps height anomalies within a local area (or, equivalently, disturbing potentials) into gravity anomalies and gravity disturbances, respectively (Prutkin and Klees 2007). In the following, we will discuss this problem in more detail, thereby restricting ourselves to the non-trivial kernel of gravity anomalies; the extension to gravity disturbances is straightforward.

The mathematical foundation of gravity field determination from gravity anomalies and geopotential numbers is the scalar Molodensky boundary-value problem (BVP). If global data are available, this problem has a non-trivial null space, which means that not all parameters of the Earth's gravity field can be uniquely determined from the given data. However, the non-uniqueness is only related to the terms of degree one of the spherical harmonic expansion of the gravity field. Taken over a local area, this non-uniqueness appears as a bias and tilt in the computed quasi-geoid, which can easily be parameterized using a low-degree polynomial. The situation changes if data are only available over a limited area of the Earth's surface, which is typical for local gravity field modelling. Then, the null space is much larger, comprising all non-zero harmonic functions that produce zero gravity anomalies over the local area. Geometric height anomalies do not suffer from any such non-uniqueness. Therefore, differences between geometric and gravimetric height anomalies also contain the part of the gravity field that cannot be modelled from terrestrial gravity data. In this context, it is the role of geometric height anomalies to determine the part of the disturbing potential (or the quasi-geoid) that cannot be determined from terrestrial gravity anomalies.

Nowadays, the contribution of functions that belong to the kernel of the gravity anomaly operator to the height anomalies may be significantly larger than the contribution from systematic errors in geometric and gravimetric height anomalies, respectively. Therefore, the following approach to combine geometric height anomalies with gravimetric height anomalies is pursued:

The differences between geometric and gravimetric height anomalies at the GPS-levelling points are used as input data to construct a non-trivial harmonic function $\tilde{T}$ that is (1) harmonic in the vicinity of the target area and (2) produces an almost zero gravity anomaly signal over the target area. Once this function has been determined, its contribution to the height anomalies is added to the gravimetric height anomalies, to give the final solution of the gravity field in terms of height anomalies. The remaining differences at the GPSlevelling points should almost reflect the influence of noise. Note that this approach not only models the contribution of the non-trivial kernel to the height anomalies, but also most systematic errors that may be present in the gravity data, GPS data, and levelling data. For more details, we refer to Prutkin and Klees (2007).

It is important to note that the algorithm of Prutkin and Klees (2007) needs information about the expected mean noise standard deviation of the differences between geometric and gravimetric height anomalies ("mean" means averaged over the target area). In practice, the standard deviation may vary over the target area, but this is not taken into account here. A possibility to account for a spatially varying standard deviation would be to split up the target area into smaller sub-areas and to apply the methodology to each sub-area individually.

If the noise standard deviation is chosen too small, the solution $\tilde{T}$ is too rough, i.e. it does not only model signal (essentially the contribution of functions that belong to the kernel of $D_{\Delta g}$ and some other systematic errors present in the data), but will also model part of the noise. If the noise standard deviation is chosen too large, the solution is too smooth and some signal will remain unmodelled.

\section{A new height reference surface for the Netherlands}

We applied our data processing strategy to compute a height reference surface for the Netherlands. All data sets are described in detail in de Min (1996) and Crombaghs and de Bruijne (2004). The main data consist of terrestrial and ship-borne point-wise free-air gravity anomalies. According to available a priori information about the standard deviation provided by Adviesdienst Geoinformatie en ICT van Rijkswaterstaat (RWS-AGI), three observation groups are formed: a group "NL" $(\sigma=0.3 \mathrm{mGal})$, which contains the gravity data on land, and two groups, "NL-IJsselmeer 1" ( $\sigma=0.6 \mathrm{mGal})$ and "NL-IJsselmeer 2" ( $\sigma=1.1 \mathrm{mGal})$, which comprise ship-borne gravity anomalies collected over the Ijsselmeer by different parties. This main data set is complemented by 14155 point-wise free-air gravity anomalies 
over Belgium, and gridded mean free-air gravity anomalies on the North-Sea (2757), Germany (2933), and France (160). They are mainly used to reduce edge effects along the border of the Netherlands.

Geometric height anomalies have been provided by RWSAGI at 82 points covering the Netherlands (cf. Fig. 1). Gravity anomalies and geometric height anomalies have been reduced for the contribution of the EIGEN-CG03C model (Förste et al. 2005). A digital terrain model has not been used, which will slightly reduce the accuracy in the Southern part of the country, where hills rise up to $300 \mathrm{~m}$ altitude. A correction for ellipsoidal effects has been applied to the gravity anomalies (cf. de Min 1996). The residual gravity anomalies, obtained from the free-air gravity anomalies after the contribution of the EIGEN-CG03C model and the ellipsoidal effects are removed, are shown in Fig. 2. They form the input data for the SRBF analysis. The use of freeair gravity anomalies is justified because the differences with the respect to the surface gravity anomalies are below $5-\mu \mathrm{Gal}$ for the Netherlands.

The Poisson kernel is used as SRBF. The mesh size of the coarse-grid SRBF network is chosen equal to $0.06^{\circ}$; the grid covers an area of $50^{\circ}-54^{\circ}$ latitude and $3^{\circ}-8^{\circ}$ longitude. The grid contains 5,628 coarse grid SRBFs, which is about $20 \%$ of all data points. The optimal depths of the coarse grid SRBFs have been found with GCV using a search interval between 2 and $40 \mathrm{~km}$ with a step size of $1 \mathrm{~km}$. Figure 3 shows the GCV functional as function of the depth. It attains the minimum at

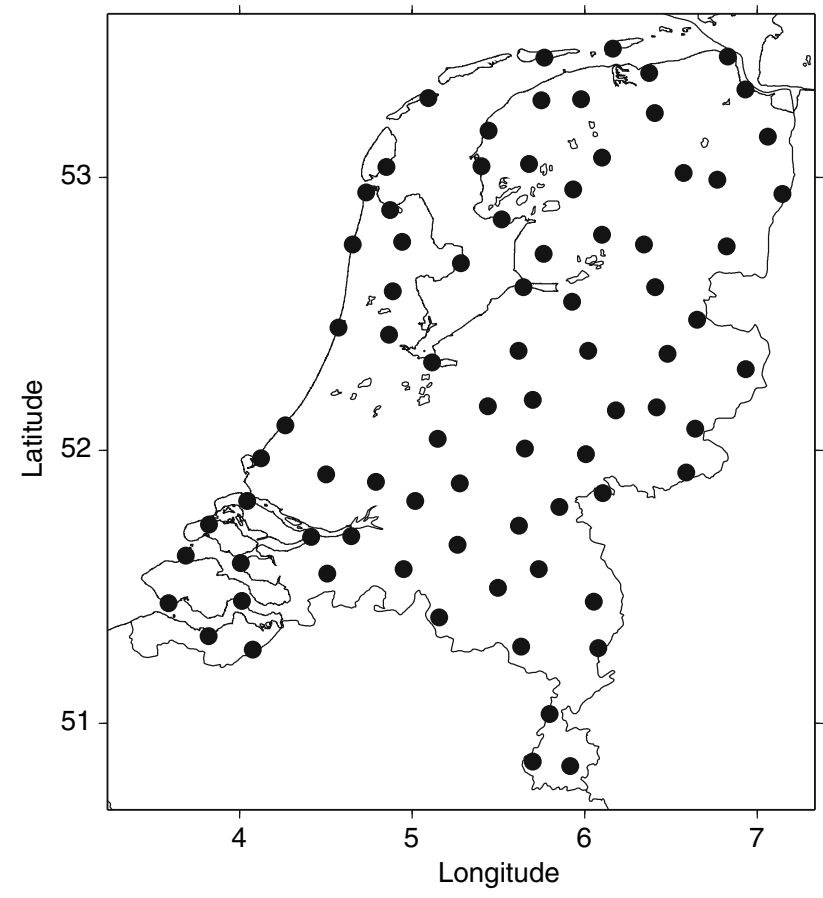

Fig. 1 Location of the 82 AGI-RWS GPS-levelling points used to compute the Dutch height-reference surface a depth of $13.5 \mathrm{~km}$, although it is quite flat between 11 and $14 \mathrm{~km}$ depth. Test computation have shown that the gravity field solution does not change much for this range of depths. The correlation length of $D_{\Delta g} \Psi$, where $\Psi$ is the Poisson kernel at $13.5 \mathrm{~km}$ depth, is about $7 \mathrm{~km}$.

The optimal depth obtained with GCV was verified by searching for the minimum RMS at 253 independent control points with observed residual gravity anomalies homogeneously distributed over the Netherlands. The search interval was left unchanged. This yielded the same depth of $13.5 \mathrm{~km}$. For the selection of the local refinement SRBFs, a threshold value for the residual of the coarse grid SRBF solution of $\tau_{1}=2.0 \mathrm{mGal}$ is used. To avoid local over-fitting, the minimum spherical distance between two SRBFs is set equal to $\psi_{\min }=0.01^{\circ}$. To avoid the modelling of large isolated residuals, a threshold of $\tau_{2}=2.0 \mathrm{mGal}$ for the average residual of the coarse grid SRBF solution in the neighbourhood of the candidate SRBF is used. The minimum number of observations within a neighbourhood of the candidate SRBF is set equal to $q=30$. GCV is used to determine the optimal depth of each local refinement SRBF, with a search interval between 0.1 and $13.5 \mathrm{~km}$ and a step size of $0.2 \mathrm{~km}$. Based on this choice for the local refinement step, 52 local refinement SRBFs have been selected within the Netherlands. They are located in the Southern part of the Netherlands close to the border to Belgium; some of them are located in the IJsselmeer region.

Figure 4 shows the residuals of the LS adjustment with 5,628 coarse grid SRBFs and 52 local refinement SRBFs. The RMS of the residuals is $0.6 \mathrm{mGal}$ for the NL data set, $0.48 \mathrm{mGal}$ for the NL-IJsselmeer 1 data set and $1.93 \mathrm{mGal}$ for the NL-IJsselmeer 2 data set. The RMS of the residuals indicates a good LS approximation of the gravity anomalies on land. An exception is the area of Limburg in the Southern part of the Netherlands. Here, we observe larger positive and negative residuals of up to $5.0 \mathrm{mGal}$. They are partly correlated with topography (see earlier on omission of terrain corrections) and partly caused by the lack of gravity data in Belgium close to the area of Limburg. Moreover, larger systematic residuals are noticeable in the ship-borne gravity anomalies of the NL-IJsselmeer 2 data set. The residuals of this data set are significantly larger than the residuals of the NL-IJsselmeer 1 data set. This is due to the lower quality of the ship-borne gravity data NL-IJsselmeer 2 compared with NL-IJsselmeer 1.

VCE has been used to estimate the standard deviation for each data set: $0.60 \mathrm{mGal}$ for the NL data set, $0.36 \mathrm{mGal}$ for the NL-IJsselmeer data set 1 , and $1.48 \mathrm{mGal}$ for the NL-IJsselmeer data set 2. The VCE estimates differ significantly from the a priori values $0.3 \mathrm{mGal}(\mathrm{NL}), 0.6 \mathrm{mGal}$ (NL-IJsselmeer 1), and $1.1 \mathrm{mGal}$ (NL-IJsselmeer 2). The VCE-values have been used as a priori values in the final LS adjustment. Then, the RMS difference between predicted 

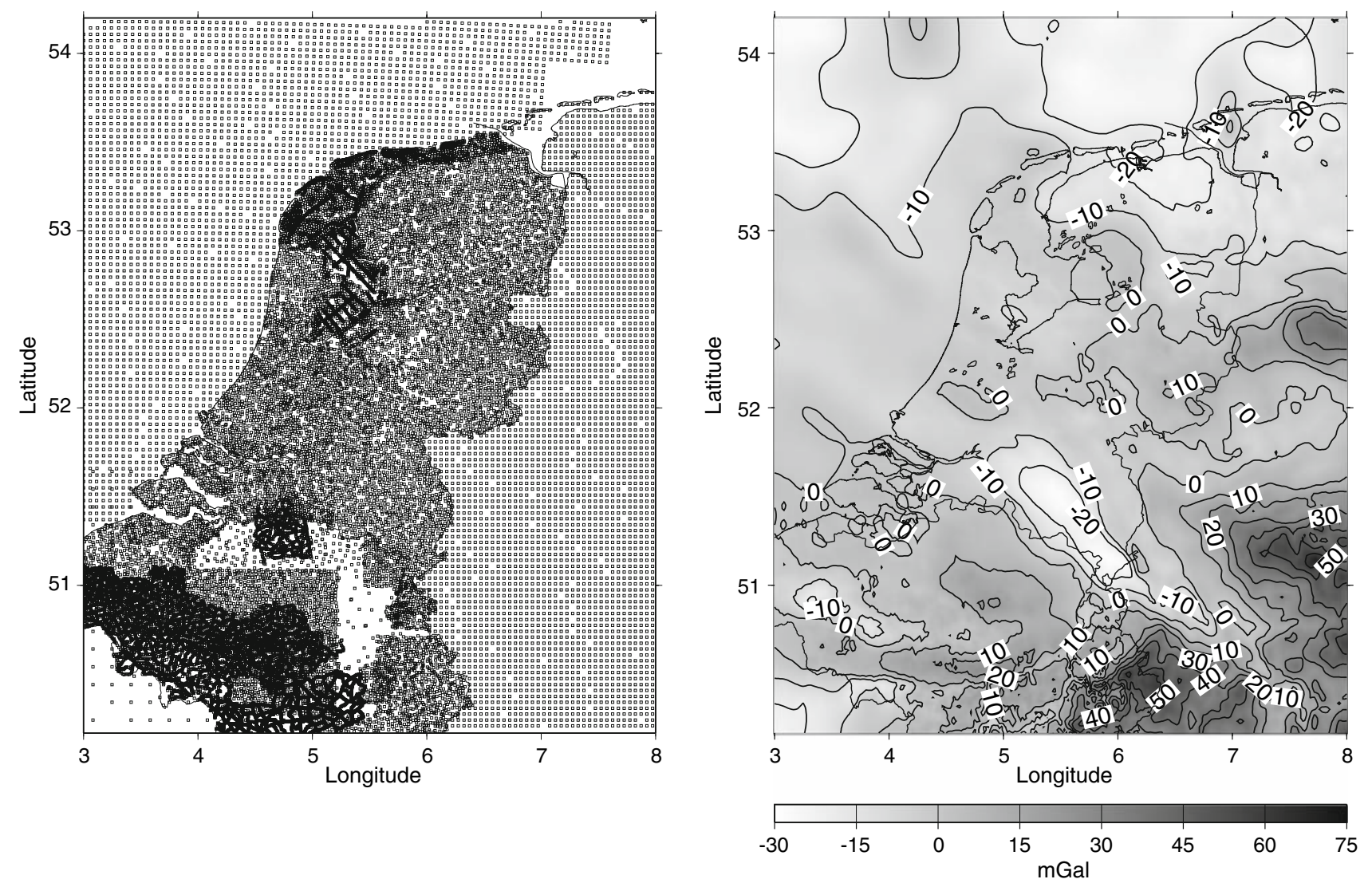

Fig. 2 Residual gravity anomalies used to compute the Dutch height-reference surface. For the Netherlands, the residual gravity anomalies range from -28.7 to $15.0 \mathrm{mGal}$; the mean value is $-5.9 \mathrm{mGal}$, and the standard deviation is $7.4 \mathrm{mGal}$

and observed gravity anomalies at the control points are $0.6 \mathrm{mGal}(\mathrm{NL}), 0.46 \mathrm{mGal}$ (NL-IJsselmeer 1) and $2.14 \mathrm{mGal}$ (NL-IJsselmeer 2). Hence, the estimated gravity field seems to be well balanced in terms of fit to the data and smoothness.

Gravimetric height anomalies are predicted at the 82 RWSAGI GPS-levelling points. Figure 5 shows the differences between the geometric height anomalies and the gravimetric height anomalies. They range between 0.310 and $0.583 \mathrm{~m}$. According to Crombaghs and de Bruijne (2004), the quality of the geometric height anomalies at the 82 RWS-AGI GPS-levelling points is about $0.010-0.015 \mathrm{~m}$. Therefore, we used a noise level of $0.01 \mathrm{~m}$ to compute the height-anomaly innovations. The latter are shown in the right panel of Fig. 5; the RMS fit to the observed height-anomaly differences is $0.7 \mathrm{~cm}$.

The sum of the SRBF model and the innovation function represents the new height reference surface, which we call "NLGEO2007". This model is compared with the official Dutch height reference surface, the NLGEO2004 (Crombaghs and de Bruijne 2004). NLGEO2004 is computed using the Stokes formula with a Meissl-Wong-Gore kernel modification (Heck and Grüninger 1988), an integration radius of

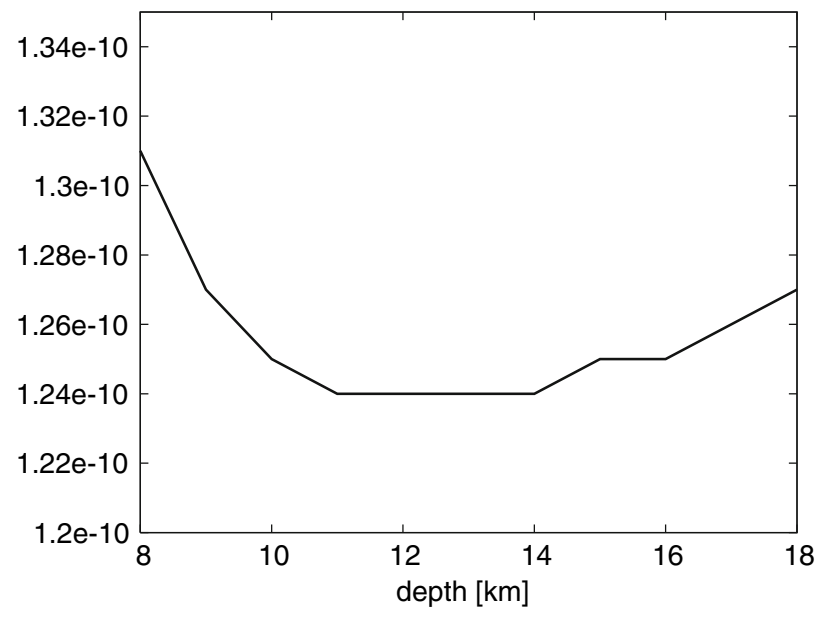

Fig. 3 The GCV functional as function of the depth of the coarse-grid SRBFs. The minimum is attained at a depth of approximately $13.5 \mathrm{~km}$

$5^{\circ}$, and a six-parameter corrector surface fitted through the 82 RWS-AGI GPS-levelling points:

$$
\begin{aligned}
\delta \zeta= & a+b\left(\lambda-5^{\circ}\right)+c\left(\varphi-52^{\circ}\right)+d\left(\lambda-5^{\circ}\right)^{2} \\
& +e\left(\varphi-52^{\circ}\right)^{2}+f\left(\lambda-5^{\circ}\right)\left(\varphi-52^{\circ}\right) .
\end{aligned}
$$




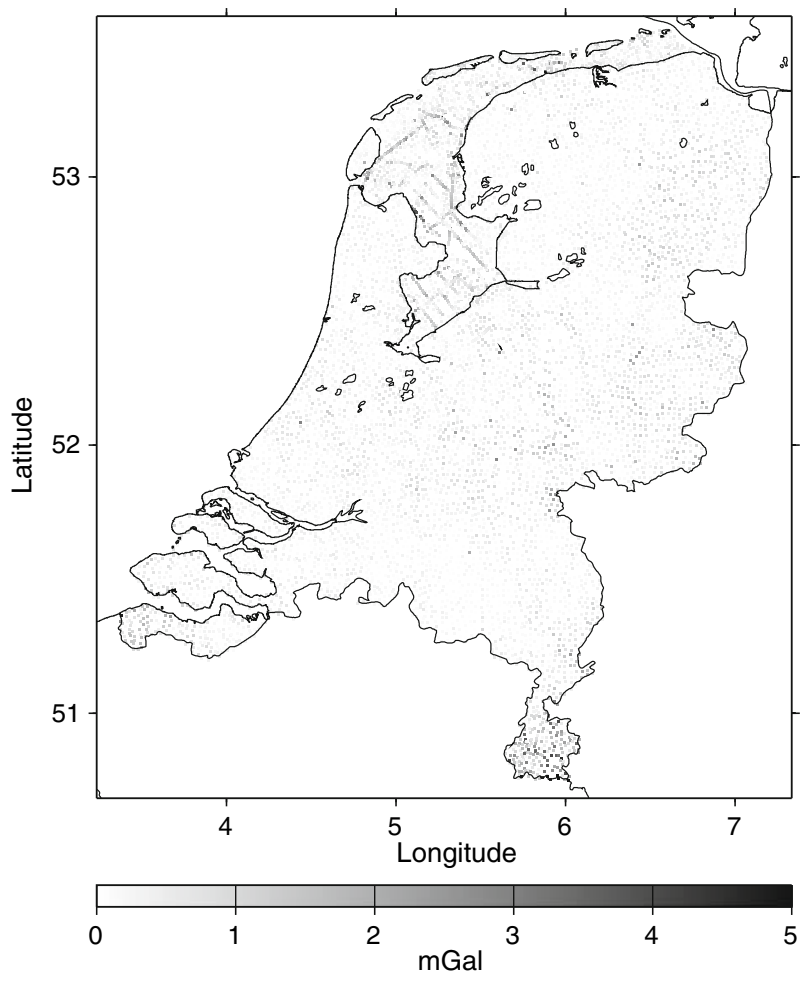

Fig. 4 Residuals of the SRBF LS adjustment using 5628 coarse grid SRBFs and 52 local refinement SRBFs. Min $=-4.8 \mathrm{mGal}$, $\max =$ $5.0 \mathrm{mGal}$, mean $=0.0 \mathrm{mGal}, \mathrm{RMS}=0.53 \mathrm{mGal}$

Table 2 shows the statistics of the residuals of NLGEO2004 and NLGEO2007, respectively, at the 82 RWS-AGI GPSlevelling points. Both models achieve about the same quality of fit. The six-parameter corrector surface, Eq. (28), performs as well as the innovation function. The reason is that the difference between geometric and gravimetric height anomalies at the 82 RWS-AGI GPS-levelling points represent a very smooth surface (see Fig. 5), and, therefore, can be easily approximated by the six-parameter corrector surface, Eq. (28).

Figure 6 shows the differences between the innovation function and the six-parameter "corrector surface". They range between -4.3 and $4.0 \mathrm{~cm}$; the RMS difference is $1.0 \mathrm{~cm}$. Prutkin and Klees (2007) have shown that for Germany, these differences vary more irregularly, which makes it difficult to find a suitable parameterization of the "corrector surface"; the approach by Prutkin and Klees (2007) solves this problem automatically.

Next, we compare NLGEO2004 and NLGEO2007 on a $1^{\prime} \times 1^{\prime}$ grid covering the Netherlands (Fig. 7). The RMS difference is $0.6 \mathrm{~cm}(\min =-1.0 \mathrm{~cm}, \max =2.7 \mathrm{~cm}$, mean $=$ $0.0 \mathrm{~cm}$ ). The differences are very small, which indicates that our approach is able to produce a high-quality height reference surface, which is competitive with NLGEO2004.

It is difficult to assess the quality of the NLGEO2004 and NLGEO2007 height reference surfaces due to the lack of

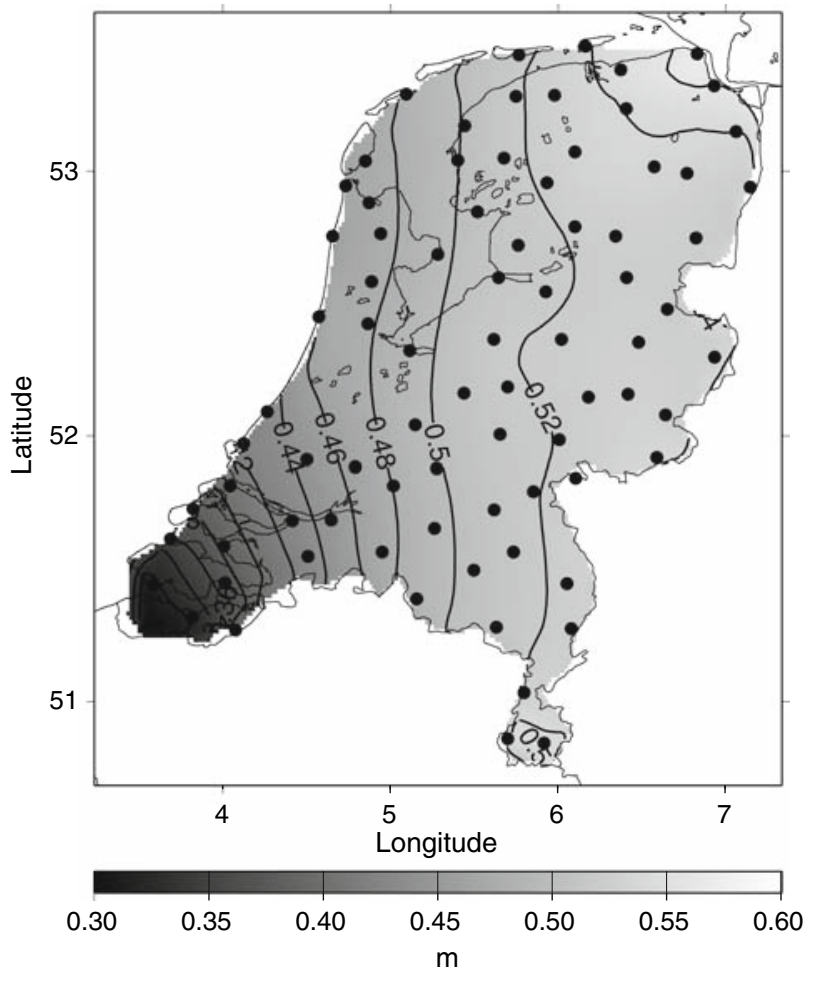

Fig. 5 The location of the 82 RWS-AGI GPS-levelling points and the height-anomaly innovations. The differences between geometric height anomalies and gravimetric height anomalies at the GPS-levelling points range from 0.310 to $0.583 \mathrm{~m}$; the mean difference is $0.488 \mathrm{~m}$, and the standard deviation is $0.053 \mathrm{~m}$. The RMS fit of the height-anomaly innovations to the given differences is $0.7 \mathrm{~cm}$

independent high-quality control data. We used 405 GPSlevelling control points, which have been provided by the Netherlands Kadaster. These data have not been used in the NLGEO2004 and NLGEO2007 solutions, because they are expected to be of lower quality. This is mainly due to the fact that the Netherlands Kadaster performs GPS measurements at these points over a time span of $2 \times 90 \mathrm{~min}$; if the ellipsoidal height difference between the two solutions is below $3 \mathrm{~cm}$, the measurements are accepted and the average value is added to the data base. In contrast, the GPS measurements at the 82 RWS-AGI GPS-levelling points lasted $24 \mathrm{~h}$. As Table 3 reveals, there are no significant differences between NLGEO2004 and NLGEO2007 at the 405 GPSlevelling control points. The RMS difference is $1.9 \mathrm{~cm}$ for the two models.

\section{Summary and conclusions}

We have developed a methodology for local gravity field modelling using SRBFs. The advantage of a SRBF representation is that (1) it can be used at the same time to represent the global and the local gravity field, (2) it allows local refinements depending on signal variation and data density, 


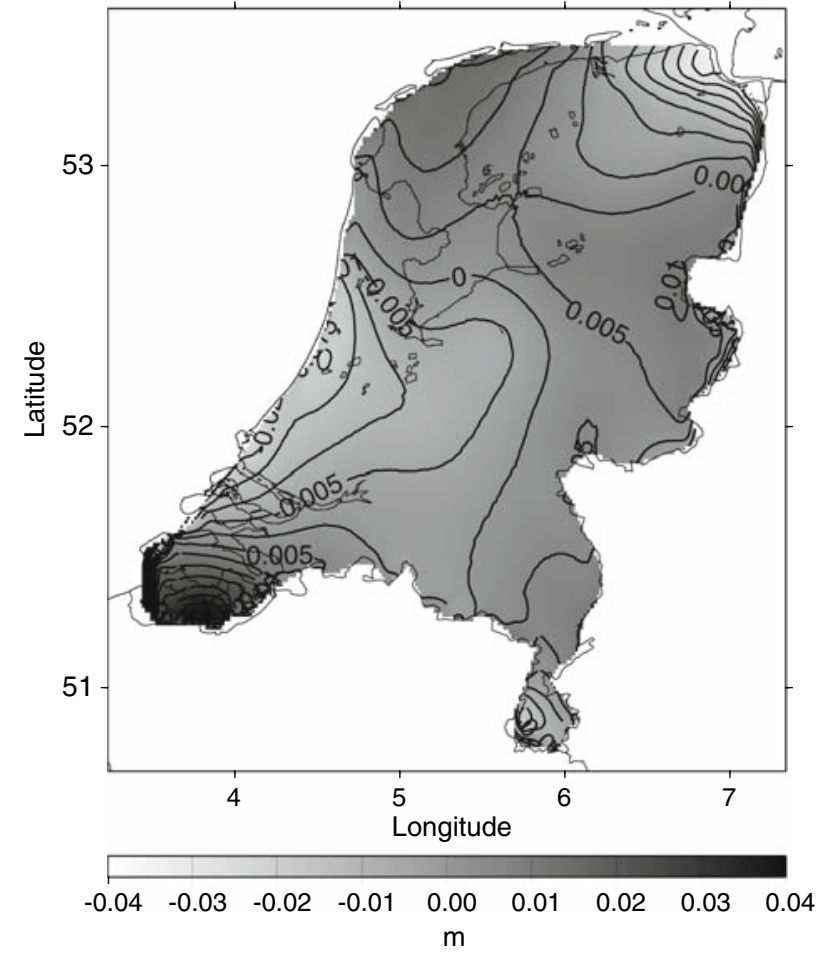

Fig. 6 Differences between the six-parameter "corrector" surface, Eq. (28), and the height-anomaly innovations. Min $=-4.3 \mathrm{~cm}, \max =$ $4.0 \mathrm{~cm}$, mean $=0.0 \mathrm{~cm}, \mathrm{RMS}=1.0 \mathrm{~cm}$

distribution, and quality, (3) it can be combined easily with an existing representation of the global gravity field in terms of spherical harmonics as every linear combination of SRBFs can directly be transformed into a spherical harmonic expansion:

$$
\begin{aligned}
T(x) & =\sum_{i=1}^{I} \beta_{i} \Psi\left(x, y_{i}\right)=\sum_{l, m} \bar{c}_{l, m}\left(\frac{R}{r}\right)^{l+1} \bar{Y}_{l, m}(\hat{x}) \Rightarrow \bar{c}_{l, m} \\
& =\psi_{l} \frac{1}{4 \pi R^{2}} \sum_{i=1}^{I} \bar{Y}_{l, m}\left(\hat{y}_{i}\right) .
\end{aligned}
$$

Therefore, a global gravity field model can be directly included in a combined LS adjustment with local gravity data. This offers a numerically simpler alternative to the joint processing of GRACE and/or GOCE level 1B data and terrestrial gravity data. Moreover, we prefer it to the traditional remove-restore technique used in local gravity field modelling, because the remove-restore technique does not make use of available information about variances and covariances of geopotential coefficients.

When working with SRBFs, the proper selection of the number and bandwidths of the SRBFs is crucial to guarantee a high-quality gravity field solution. The developed algorithms selects the number and the optimal bandwidths automatically, depending on signal variation, data distribution, and data accuracy. This helps avoiding over- and

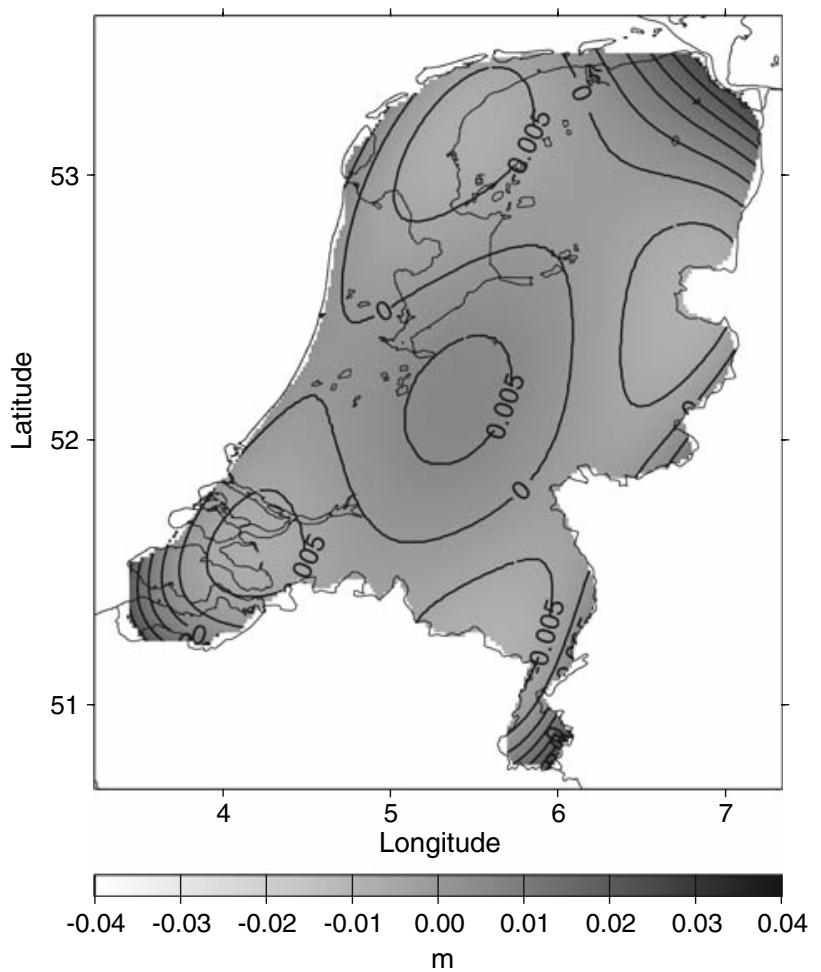

Fig. 7 Differences between the official Dutch height-reference surface NLGEO2004 and the NLGEO2007. $\mathrm{Min}=-1.0 \mathrm{~cm}, \max =2.7 \mathrm{~cm}$, mean $=0.0 \mathrm{~cm}$, RMS $=0.6 \mathrm{~cm}$

under-parameterization and guarantees a smooth gravity field solution. The numerical efficiency of the selection procedure is superior to alternative procedure proposed in the past (e.g. Barthelmes 1986; Marchenko et al. 2001) due to the twostep procedure of coarse grid solution and local refinement. Whether this is really an advantage in practical applications depends on the available computer hardware. The price to be paid for the improved numerical efficiency is a slightly higher number of SRBFs compared with the approach by Marchenko et al. (2001) (see also Klees et al. 2005).

Our methodology foresees two different approaches of using GPS-levelling data: (1) the direct approach, which means that GPS-levelling data are added to the functional model and processed together with the gravity data in a LS adjustment (this approach has not been addressed in this paper), and (2) an indirect approach (Prutkin and Klees 2007). The latter interprets the differences between gravimetric height anomalies and geometric height anomalies as the contribution of the non-trivial kernel of the gravity-anomaly operator, which leads to the solution of a Cauchy BVP. This approach is numerically more complicated than the direct approach, but takes into account the physical nature of the differences between geometric and gravimetric height anomalies. Compared with the more traditional approach of using a "corrector surface", it has the additional advantage that the 
Table 2 Residual differences of NLGEO2004 and NLGEO2007, respectively, at the 82 RWS-AGI GPS-levelling points

\begin{tabular}{llllll}
\hline Model & GPS-levelling points & RMS $(\mathrm{cm})$ & $\min (\mathrm{cm})$ & $\max (\mathrm{cm})$ & 2.4 \\
\hline NLGEO2004 & 82 RWS-AGI & 0.7 & -1.5 & 1.1 & 0.0 \\
NLGEO2007 & 82 RWS-AGI & 0.7 & -1.3 & 0.0 \\
\hline
\end{tabular}

Table 3 Differences between NLGEO2004 and NLGEO2007, respectively, and geometric height anomalies at 405 independent GPS-levelling control points provided by the Dutch Kadaster

\begin{tabular}{llllrr}
\hline Model & GPS-levelling points & RMS $(\mathrm{cm})$ & $\min (\mathrm{cm})$ & $\max (\mathrm{cm})$ & mean $(\mathrm{cm})$ \\
\hline NLGEO2004 & 405 Kadaster & 1.9 & -8.2 & 11.0 & 0.2 \\
NLGEO2007 & 405 Kadaster & 1.9 & -9.2 & 9.1 & 0.0 \\
\hline
\end{tabular}

user does not have to make any decision regarding the proper choice of the model.

Compared with LSC, which provides in fact a minimumnorm SRBF solution, the proposed methodology for the processing of the gravity data offers more flexibility. LSC uses SRBFs with the same bandwidth, which is determined by the correlation length of the gravity field, whereas our approach permits SRBFs with different bandwidths, and selects them automatically from the data. In combination with the coarse grid and local refinement step, this reduces the number of SRBFs and may provide a better adaptation to the data in the presence of local non-isotropy.

We found empirically that the number of SRBFs is usually less than $25 \%$ of the number of data points. LSC uses formally as many SRBFs as data points, although in practice often a subset of the data points is used either to reduce the numerical complexity or because the data contain redundant information. However, in LSC the selection of the subset is up to the user and no objective measures appear to be used to do this. We also want to mention that LSC is the minimum-norm solution in the reproducing kernel Hilbert space of SRBFs, whereas our solution is a discrete LS solution, which lacks convergence if the data density goes to infinity.

The methodology has been applied to compute a new height-reference surface for the Netherlands. A comparison with the official Dutch height-reference surface has shown that our solution has at least the same quality as the official one. A more detailed analysis of the quality of the two heightreference surfaces requires high quality GPS-levelling data, which are not available yet.

There is some scope for further improvements of the proposed methodology. For instance, the combination of gravimetric quasi-geoid with GPS-levelling points according to Prutkin and Klees (2007) requires information about the accuracy of the gravimetric quasi-geoid and the GPSlevelling data. So far, only a single variance factor for the complete data set can be taken into account. If the accuracy is spatially non-homogeneous, the application of a single ('average') variance factor could lead to overfit in one subarea and to underfit in another sub-area. A partial solution to this problem could be to subdivide the area into sub-areas of "homogeneous" accuracy and to process each sub-are a separately. Said earlier, sometimes full variance-covariances are available for individual data sets; this information cannot be easily combined with the proposed data combination strategy based on the formulation as a Cauchy boundary-value problem. Then, a generalized LSC approach as proposed by Kotsakis and Sideris (1999) and applied by Fotopoulos et al. (2003) may be appropriate.

Acknowledgments The Adviesdienst Geoinformatie en ICT of Rijkswaterstaat (RWS-AGI) provided the gravity data sets and the 82 GPSlevelling points. Netherlands Kadaster provided the 405 GPS-levelling points. This support is gratefully acknowledged. We are very thankful to the EiC Will Featherstone, the responsible editor Nico Sneeuw, the reviewers Frans Barthelmes and Martin Vermeer, and an anonymous reviewer for their very valuable comments, which helped us to improving the manuscript.

Open Access This article is distributed under the terms of the Creative Commons Attribution Noncommercial License which permits any noncommercial use, distribution, and reproduction in any medium, provided the original author(s) and source are credited.

\section{References}

Barthelmes F (1986) Untersuchungen zur Approximation des äusseren Gravitationsfeldes der Erde durch Punktmassen mit optimierten Positionen. Veröffentlichungen des Zentralinstituts für Physik der Erde, Nr. 92, Potsdam

Barthelmes F (1988) Local gravity field approximation by point masses with optimized positions. In: Proc. 6th international symposium "Geodesy and Physics of the Earth", Potsdam

Bjerhammar A (1976) A Dirac approach to physical geodesy. Zeitschrift für Vermessungswesen (ZfV) 101:41-44

Blackman RB, Tukey JW (1958) The measurement of power spectra from the point of view of communication engineering-part II. Bell Syst Tech J485-569 
Blaha G, Blessette RP, Hadgigeorge G (1986) Global point-mass adjustment of the oceanic geoid based on satellite altimetry. Mar Geodesy 10:97-129

Bottoni GP, Barzaghi R (1993) Fast collocation. Bulletin Géodésique 67:119-126

Chambodut A, Panet I, Mandea M, Diament M, Holschneider M, Jamet O (2005) Wavelet frames: an alternative to spherical harmonic representation of potential fields. Geophys J Int 163:875-899

Crombaghs M, de Bruijne A (2004) NLGEO2004 - een nieuw geoïdemodel voor Nederland. Adviesdienst Geo-informatie en ICT, Report AGI-GAP-2004-25, Delft, $41 \mathrm{p}$

Eicker A, Mayer-Gürr T, Ilk KH (2004) Global gravity field solutions from GRACE SST data and regional refinements by GOCE SGG observations. In: Proceedings IAG international symposium gravity, geoid and space missions (GGSM2004), Porto, Portugal

Förste C, Flechtner F, Schmidt R, Meyer U, Stubenvoll R, Barthelmes F, König R, Neumayer KH, Rothacher M, Reigber Ch, Biancale R, Bruinsma S, Lemoine JM, Raimondo JC, (2005) A new high resolution global gravity field model derived from combination of GRACE and CHAMP mission and altimetry/gravimetry surface gravity data. Poster presented at EGU General Assembly 2005, Vienna

Förstner W (1979) Ein Verfahren zur Schätzung von Varianz- und Kovarianzkomponenten. Allgemeine Vermessungsnachrichten $86: 446-453$

Fotopoulos G, Kotsakis C, Sideris MG (2003) How accurately can we determine orthometric height differences from GPS and geoid data. J Surv Eng 1:1-10

Freeden W, Reuter R (1983) Spherical harmonic splines: theoretical and computational aspects. Meth u Verf d Math Phys 27:79103

Freeden W, Schreiner M, Franke R (1997) A Survey on spherical spline approximation. Surv Math Ind 7:29-85

Freeden W, Gervens T, Schreiner M (1998) Constructive approximation on the sphere (with applications to geomathematics). Oxford Science Publication, Oxford University Press, New York

Golub GH, Heath M, Wahba G (1979) Generalized cross-validation as a method for choosing a good ridge parameter. Technometrics 21:215-223

Hardy RL, Göpfert WM (1975) Least squares prediction of gravity anomalies, geoidal undulations, and deflections of the vertical with multiquadric harmonic functions. Geophys Res Lett 2:423-426

Heck B, Grüninger W (1988) Modification of Stokes' integral formula by combining two classical approaches. In: Proceedings IAG symposia, XIX IUGG general assembly, Vancouver/Canada, 10-22 August 1987, Paris 1988, Tome II, pp 319-337

Heck B, Seitz K (1993) Effects on non-linearity in the geodetic boundary value problem. Deutsche Geodätische Kommission, Reihe A, Heft Nr. 109, München

Heck B, Seitz K (2003) Solutions of the linearized geodetic boundary value problem for an ellipsoidal boundary to order $e^{3}$. J Geod 77:182-192

Heikkinen M (1981) Solving the shape of the Earth by using digital density models. Finnish Geodetic Institute, Report 81:2, Helsinki

Holschneider M, Iglewska-Nowak I (2007) Poisson wavelets on the sphere. J Fourier Anal Appl 13:405-419

Holschneider M, Chambodut A, Mandea M (2003) From global to regional analysis of the magnetic field on the sphere using wavelet frames. Phys Earth Planet Inter 135:107-124

Klees R, Marchenko NK, Alberts B, Wittwer T (2005) Comparison of various methods for the inversion of airborne gravity data. In: Proceedings dynamic planet 2005, Cairns, Australia (CD-ROM)

Klees R, Wittwer T (2007a) A data-adaptive design of a spherical basis function network for gravity field modelling. In: Tregoning $\mathrm{P}$, Rizos C (eds) Dynamic planet-monitoring and understanding a dynamic planet with geodetic and oceanographic tools, interna- tional association of geodesy symposia, vol 130. Springer, Berlin, pp 323-328

Klees R, Wittwer T (2007b) Local gravity field modelling with multipole wavelets. In: Tregoning P, Rizos C (eds) Dynamic planetmonitoring and understanding a dynamic planet with geodetic and oceanographic tools. In: International association of geodesy symposia, vol 130. Springer, Berlin, pp 303-308

Koch KR, Kusche J (2002) Regularization of geopotential determination from satellite data by variance components. J Geod 76:259268

Kotsakis C, Sideris MG (1999) On the adjustment of combined GPSlevelling/geoid networks. J Geod 73:412-421

Krarup T (1969) A contribution to the mathematical foundation of physical geodesy. Report 44, Danish Geodetic Institute, Copenhagen

Kusche J (2003) A Monte-Carlo technique for weight estimation in satellite geodesy. J Geod 76:641-652

Kusche J, Ilk KH, Rudolph S, Thalhammer M (1998) Application of spherical wavelets for regional gravity field recovery-a comparative study. In: Forsberg R, et al (ed) Geodesy on the Move, IAG symposia, vol 119. Springer, Berlin, pp 213-218

Lelgemann D (1981) On numerical properties of interpolation with harmonic kernel functions. Manuscr Geod 6:157-191

Lelgemann D, Marchenko AN (2001) On concepts for modelling the Earth's gravity field. Deutsche Geodätische Kommission, Reihe A, Heft Nr. 117, München

Marchenko AN (1998) Parametrization of the Earth's gravity field: point and line singularities. Lviv Astronomical and Geodetical Society, Lviv

Marchenko AN (2003) Regional gravity field model from satellite altimetry, gravimetry and GPS-levelling data in the Black Sea area. Bollettino Di Geodesia E Scienze Affini 1XII:245-259

Marchenko AN, Barthelmes F, Meyer U, Schwintzer P (2001) Regional geoid determination: an application to airborne gravity data in the Skagerrak. Scientific Technical Report no. 01/07, GeoForschungsZentrum Potsdam, $50 \mathrm{pp}$

de Min E (1996) The geoïde voor Nederland. Nederlands Commissie voor Geodesie, Delft, $224 \mathrm{p}$

Panet I, Chambodut A, Diament M, Holschneider M, Jamet O (2006) New insights on intraplate volcanism in French Polynesia from wavelet analysis of GRACE, CHAMP, and sea surface data. J Geophys Res 111:B09403. doi:10.1029/2005JB004141

Prutkin I, Klees R (2007) The non-uniqueness of local quasi-geoids computed from terrestrial gravity anomalies. J Geod. doi:10.1007/ s00190-007-0161-1

Reilly JP, Herbrechtsmeier EH (1978) A systematic approach to modelling the geopotential with point mass anomalies. J Geophys Res $83: 841-844$

Sanso F, Tscherning CC (2003) Fast spherical collocation: theory and examples. J Geod 77:101-112

Schmidt M, Fabert O, Shum CK, Han SC (2004) Gravity field determination using multiresolution techniques. Proceedings 2nd international GOCE User workshop, ESA-ESRIN, Frascati

Schmidt M, Kusche J, van Loon J, Shum CK, Han SC, Fabert O (2005) Multi-resolution representation of regional gravity data. In: Jekeli C, Bastos L, Fernandes J (eds) Gravity, geoid and space missions. Springer, Berlin, pp 167-172

Schmidt M, Fengler M, Mayer-Gürr T, Eicker A, Kusche J, Sanchez L, Han SC (2007) Regional gravity modelling in terms of spherical base functions. J Geod 81:17-38

Sünkel H (1981) Point mass models and the anomalous gravitational field. Report 328, Department of Geodetic Sciences, Ohio State University, Columbus

Tenzer R, Klees R (2007) The choice of the spherical radial basis functions in local gravity field modelling. Studia Geophys Geod (in review) 
Tscherning CC (1986) Functional methods for gravity field approximation. In: Sünkel H (ed) Mathematical and numerical techniques in physical geodesy, Lecture Notes in earth sciences, vol 7. Springer, Berlin, pp 3-47

Vermeer M (1982) The use of mass point models for describing the Finnish gravity field. Proc. 9th meeting of the Nordic Geodetic Commission, Gävle, Sweden
Vermeer M (1983) A new SEASAT altimetric geoid for the Baltic. Finnish Geodetic Institute, Report 83:4, Helsinki

Vermeer M (1984) Geoid studies on Finland and the Baltic. Finnish Geodetic Institute, Report 84:3, Helsinki

Vermeer M (1995) Mass point geopotential modelling using fast spectral techniques; historical overview, toolbox description, numerical experiment. Manuscr Geod 20:362-378 